\title{
Ewing's sarcoma in the spinal canal of T12-L3: A case report and review of the literature
}

\author{
DAJUN YAN $^{1}$, JIE ZHANG $^{2}$ and DEQUAN ZHONG ${ }^{1}$ \\ ${ }^{1}$ Neurosurgical Research Institute, The First Affiliated Hospital of Guangdong Pharmaceutical University, \\ Guangzhou, Guangdong 510060; ${ }^{2}$ Department of Epidemiology, School of Basic Medicine, \\ Jinan University, Guangzhou, Guangdong 510000, P.R. China
}

Received March 9, 2019; Accepted September 11, 2019

DOI: $10.3892 / 01.2019 .10958$

\begin{abstract}
Primary Ewing's sarcoma (ES) is rare, especially when it occurs in the spinal canal during middle or old age. The rarity of Ewing's sarcoma breakpoint region 1 fusion-negative ES has been reported in the literature. The present case report describes a 60-year-old Chinese patient who was diagnosed with ES originating from the spinal canal in 2016. The patient was hospitalized with pain resembling electric shock in the waist and buttocks, which occurred intermittently for 1 month, and incontinence for 1 week. Magnetic resonance imaging demonstrated multiple inhomogeneous, oval-shaped nodules in the intradural and cauda equina spaces of T12-L3. The largest nodule was $\sim 23 \times 11 \times 10 \mathrm{~mm}$. The patient underwent right adrenal tumour resection. A histopathologic examination of the focal area revealed that the tumour consisted of small, circular haematoxylin stained cells that formed typical Homer-Wright rosettes. Immunohistochemical analysis confirmed that the patient suffered from ES due to positive staining for membranous cluster of differentiation 99 (CD99), cytokeratin (CK) and nuclear foetal-liver infusion 1 (FLI-1). In conclusion, the histopathological presence of Homer-Wright rosettes and immunohistochemical markers such as CD99, FLI-1 and CK are valuable factors for the diagnosis of ES, although cytogenetic analysis is considered the gold standard. Complete surgery is the most effective treatment option for ES treatment. Adjuvant radiotherapy
\end{abstract}

Correspondence to: Dr Dequan Zhong, Neurosurgical Research Institute, The First Affiliated Hospital of Guangdong Pharmaceutical University, 19 Nonglin Xia Road, Guangzhou, Guangdong 510060, P.R. China

E-mail: 13668900049@163.com

Abbreviations: ES, Ewing's sarcoma; $\mathrm{CD}$, cluster of differentiation; FLI-1, foetal-liver infusion 1; CK, cytokeratin; MRI, magnetic resonance imaging

Key words: Ewing's sarcoma, spinal canal, diagnosis, treatment, cluster of differentiation 99, foetal-liver infusion 1 and combination chemotherapy can also improve the survival rate of patients postoperatively.

\section{Introduction}

Ewing's sarcoma (ES) commonly affects the growth of metaphyseal bones. Although primary ES of the spine is rare (1), it is commonly observed in the sacrum. The highest incidence of ES is observed in patients in their 20s, and mostly involves the long bones and the pelvis. Spinal ES is divided into two groups: i) The sacral type, which includes the sacrum and coccyx, the incidence of which is $<5 \%$ (2) and ii) the non-sacral type, which includes the cervical, dorsal and lumbar vertebrae (3). The incidence of non-sacral ES is $>0.9 \%$. In the majority of cases, vertebrae are affected following the metastasis of ES, which originates elsewhere. It is very rare to encounter primary vertebral ES if the sacrum is excluded. Surgery combined with chemotherapy and radiotherapy to control the progression of neurological deficits is associated with a preferable outcome (3). The treatment of ES is challenging, and there is currently no global uniform treatment standard. The present report describes a case of a middle aged patient with multiple ES in the spinal canal of T12-L3 who was admitted to the First Affiliated Hospital of Guangdong Pharmaceutical University (Guangzhou, China) in November 2016.

\section{Case report}

A 60-year-old male Chinese patient presented with pain resembling electric shock in the waist and buttocks, which occurred intermittently for 1 month, and incontinence for 1 week. A neurophysical examination demonstrated weakness of the lower extremities (power grade IV/V), decreased sensation below the ankle joint (the right side was more affected compared with the left side), two-sided knee tendon hyperreflexia and a positive Babinski sign. Magnetic resonance imaging (MRI) is a medical imaging technique used in radiology to form pictures of the anatomy and the physiological processes of the body (4). MRI of the patient revealed multiple inhomogeneous, oval-shaped nodules in the intradural and cauda equina spaces of T12-L3. The largest nodule was $\sim 23 \times 11 \times 10 \mathrm{~mm}$, with a high signal in T2-weighted, T2 spectral presaturation with inversion recovery and T1-weighted imaging (Fig. 1), which was 
inhomogeneously reinforced following gadolinium injection (Fig. 2). The oedema signal of T1 and T2 was strip-like in the adjacent thoracic cord. The preoperative diagnosis was spinal cord or cauda equina occupying lesions, considering multiple metastases of the cauda equina in the spinal canal of T12-L3. The patient underwent a T12-L3 laminectomy. Opening of the dura mater revealed an expansion of the conus medullaris and two non-enveloped, soft, greyish-white occupied lesions of the cauda equina (Fig. 3). The occupied lesions were partly abscised using microsurgical tools. Sarcoma was diagnosed with frozen section analysis while the surgery was being performed. The nerve tracts were saved, and the remaining occupied lesions were completely removed (Fig. 4). Opening of the white expansion of the conus medullaris revealed soft, greyish-brown tissue (Fig. 5). The tissue was removed using microsurgical tools, and the histopathological results were consistent with ES. Postoperative MRI did not detect the existence of a remaining tumour. Soft tissue swelling was presented after surgery (Fig. 6). Immunohistochemistry of the tumour revealed hypercellular areas, tissue with monomorphic small blue circular cells lacking cytoplasm with focal clearing of the cytoplasm arranged in sheets and compact nest patterns (Fig. 7). The tumour cells were not arranged in well-formed rosettes. The round-to-oval nuclei had finely distributed chromatin and small nucleoli. Mitotic figures were infrequent. Additionally, foci of necrosis, haemorrhage and oedema within the tumour were observed. Immunohistochemistry was performed on tissue sections to investigate the presence of certain proteins in tumour cells. The antibodies (OriGene Technologies, Inc.) used in the immunohistochemistry experiment were monoclonal antibodies secreted by a B lymphocyte clone. The immunohistochemical analysis revealed positivity for cluster of differentiation 99 (CD99; clone PCB1) (Fig. 8), foetal-liver infusion 1 (FLI-1; clone MRQ-1) (Fig. 9), cytokeratin (CK; clone AE1/AE3) (Fig. 10), negativity for CD34, CD20, CD3, SOX-10, glial fibrillary acidic protein, progesterone receptor, $\alpha$-fetoprotein, CD117, placental alkaline phosphatase, synapsin, glycoprotein hormone $\alpha$ chain, prostate-specific antigen, napsin A, transcription termination factor 1 , interleukin-12 subunit $\beta$ and tumour protein $\mathrm{P} 63$, and $80 \%$ of the cells were positive for $\mathrm{Ki}-67$, which supported the diagnosis. Based on the patient's positive immunohistochemistry results, an MRI at the corresponding position was performed to identify metastatic tumors and primary lesions. No suspicious lesions were identified in the patient's brain, lungs or prostate (Fig. 11). Fluorescence in situ hybridization (FISH) is a molecular cytogenetic technique that uses fluorescent probes that bind to the parts of a nucleic acid sequence with a high degree of sequence complementarity; fluorescence microscopy can be used to detect the fluorescent probe bound to the chromosomes $(5,6)$. Genetic FISH analysis of the Ewing's sarcoma breakpoint region 1 (EWSR1) gene in 200 interphase cells from the patient demonstrated no specific cytogenetic abnormalities. Each signal pattern exhibited the following: $2 \mathrm{~F}, 32.0 \%$; $1 \mathrm{~F}, 10 \%$; $3 \mathrm{~F}, 45.0 \%$; $4 \mathrm{~F}, 4.0 \%$; $1 \mathrm{G} 1 \mathrm{R} 1 \mathrm{~F}$, $2.5 \%$; $1 \mathrm{G} 1 \mathrm{R} 2 \mathrm{~F}, 2.0 \%$ and $5 \mathrm{~F}, 4.5 \%$ (F, G and R stand for fusion, green and red, respectively; Fig. 12). FISH analysis shows fusion cell $<10 \%$, indicating that the specimen was considered FISH-negative. Adjuvant chemotherapy was suggested; the patient accepted radical surgery followed by combination chemotherapy, but the disease continued to progress. Following
A

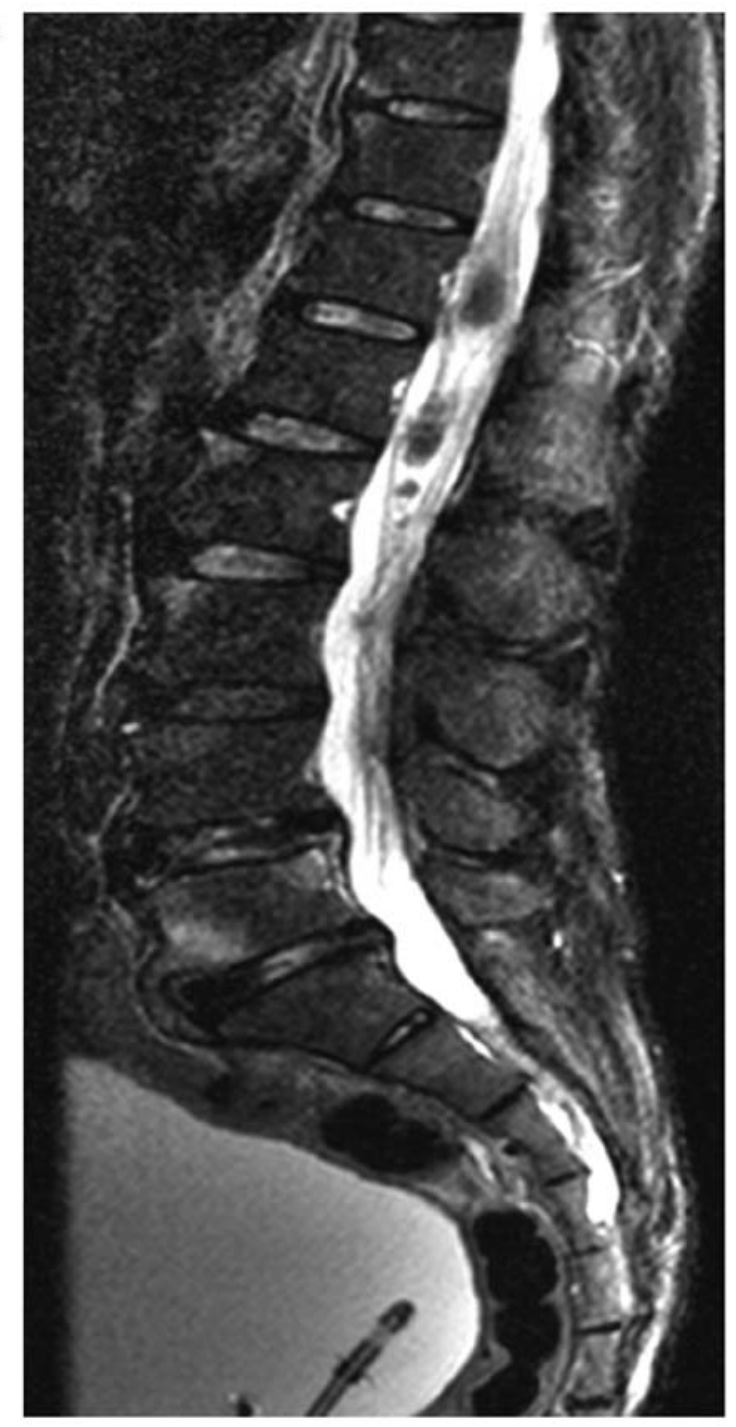

B

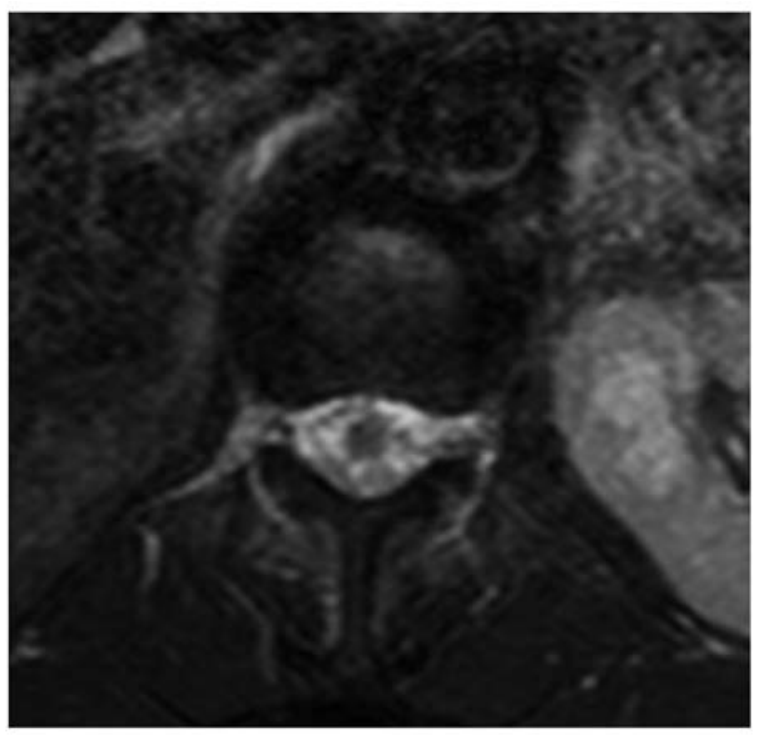

Figure 1. Preoperative T2 spectral presaturation with inversion recovery images. (A) Sagittal section and (B) cross-section.

chemotherapy treatment, the patient suffered from depression and refused any further treatment; the patient was lost to follow-up as they did not return. 
A

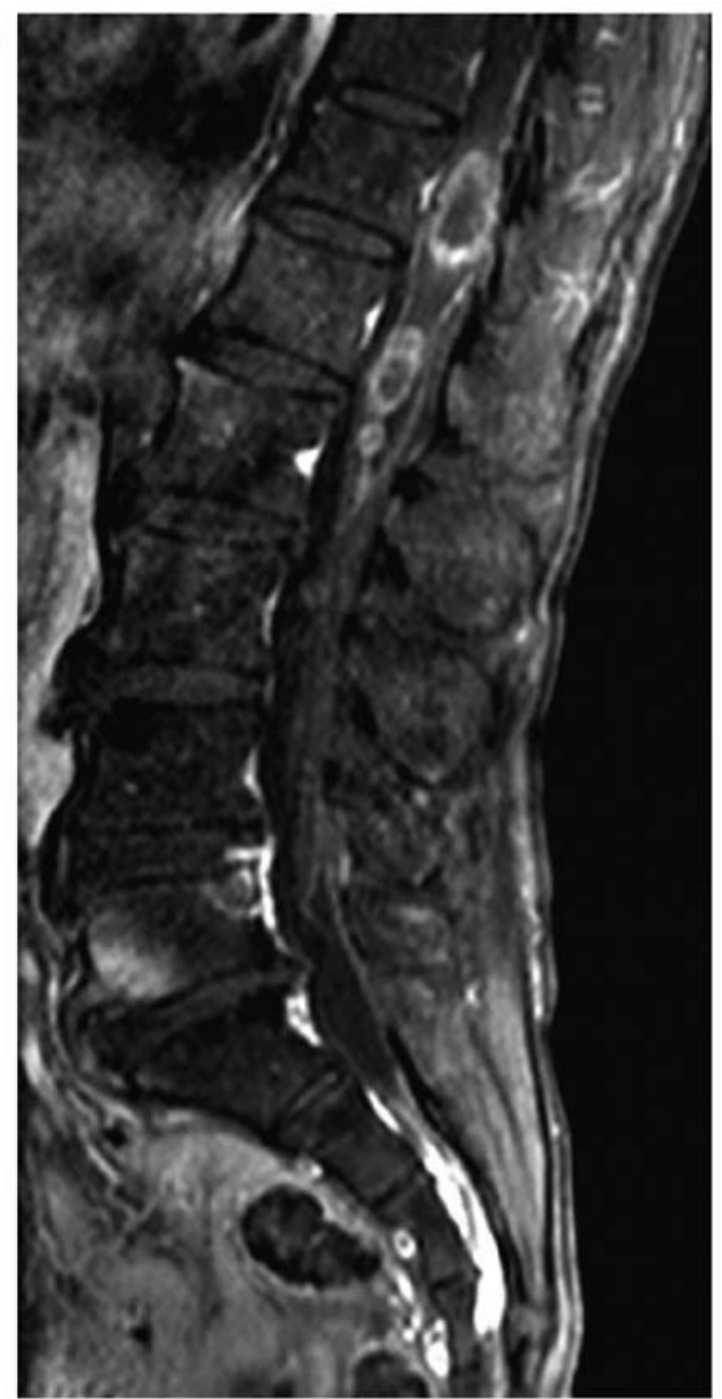

B

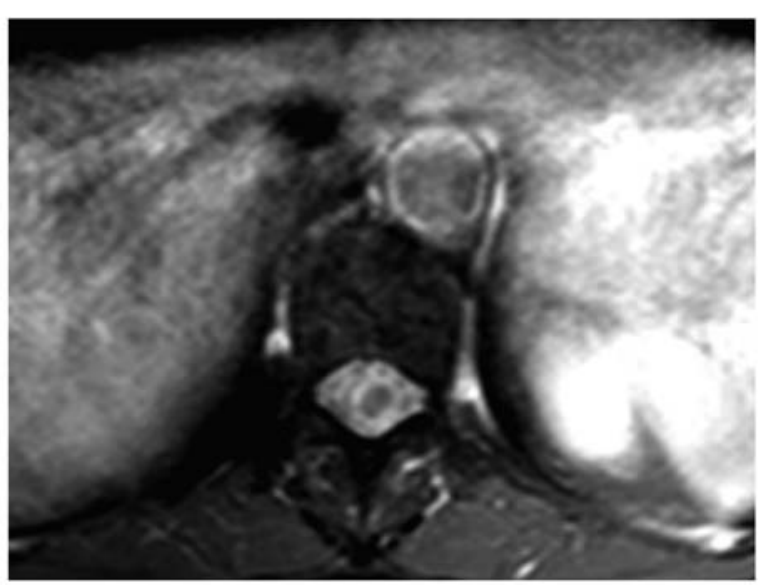

Figure 2.Preoperative enhancement. (A) Sagittal section and (B) cross-section. T12-L3 exhibited multiple inhomogeneous, oval-shaped nodules. The largest nodule was $\sim 23 \times 11 \times 10 \mathrm{~mm}$.

\section{Discussion}

ES is a developmental tumour characterized by balanced chromosomal translocations and the formation of new fusion genes. ES is an aggressive tumour with high occurrence of metastasis in children and young teenagers, and is caused by chromosomal

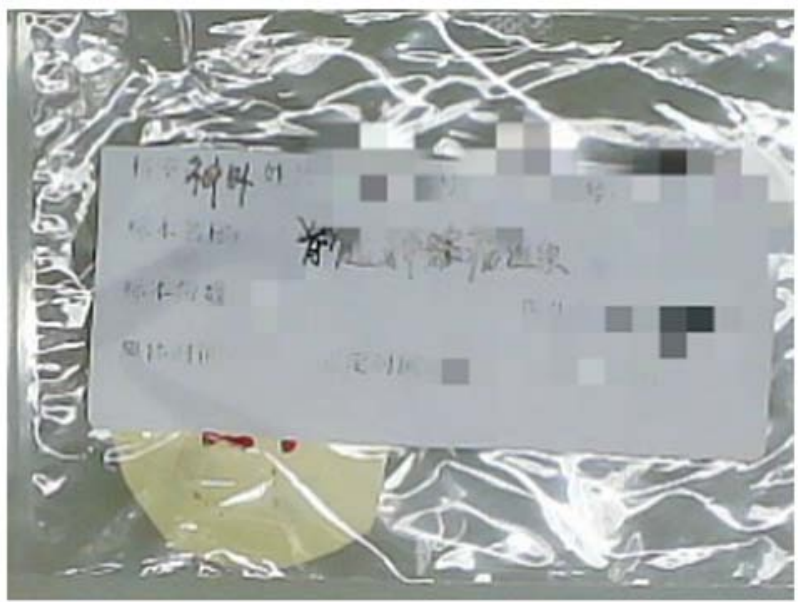

Figure 3. Intraoperative pathological examination: Nonenveloped, soft, greyish-white occupied lesions of the cauda equina.

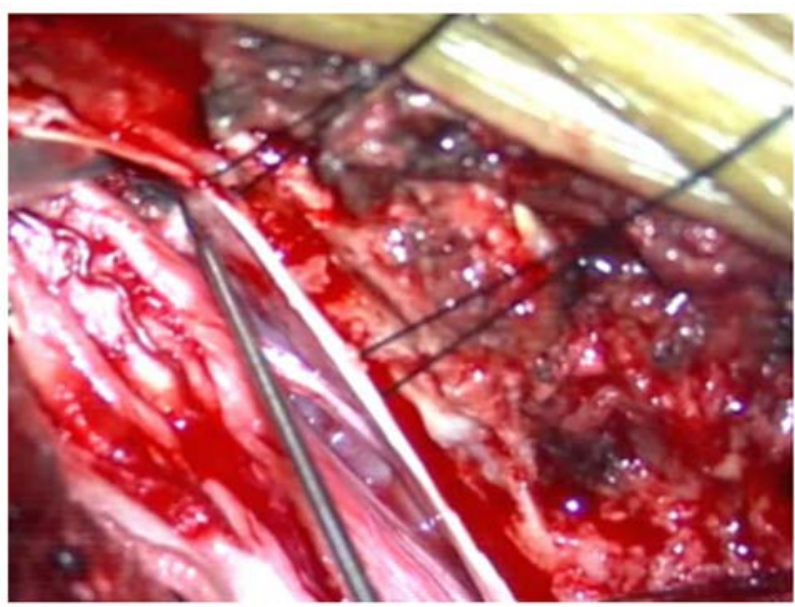

Figure 4. Intraoperative image. After the sarcoma was diagnosed by frozen section analysis during the surgery, the nerve tracts were saved and the remaining occupied lesions were completely removed.

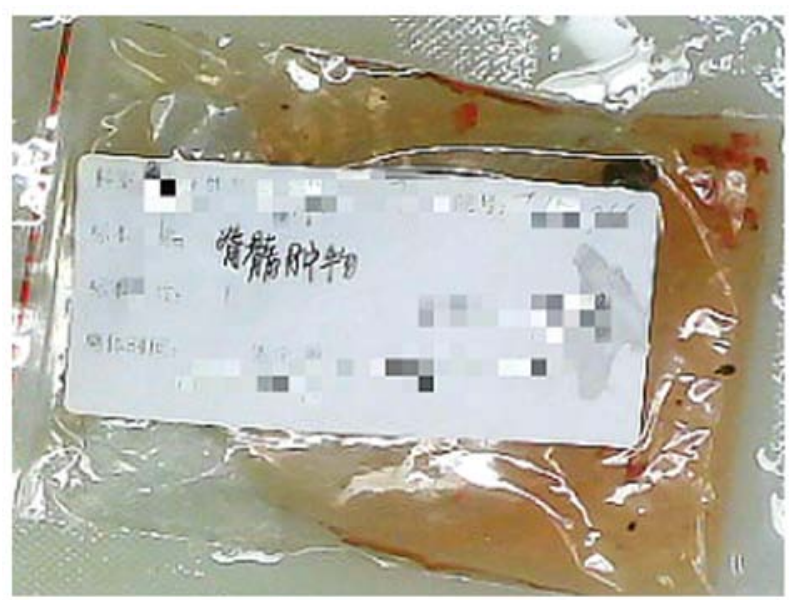

Figure 5. Postoperative pathological examination. Opening of the white expansion of the conus medullaris revealed soft, greyish-brown tissue.

fusion in EWSR1 genes (7). ES can affect any bone, but mostly affects the lower extremities (45\%), followed by the pelvis (20\%), upper extremities (13\%), axial skeleton and ribs (13\%) or face 
A

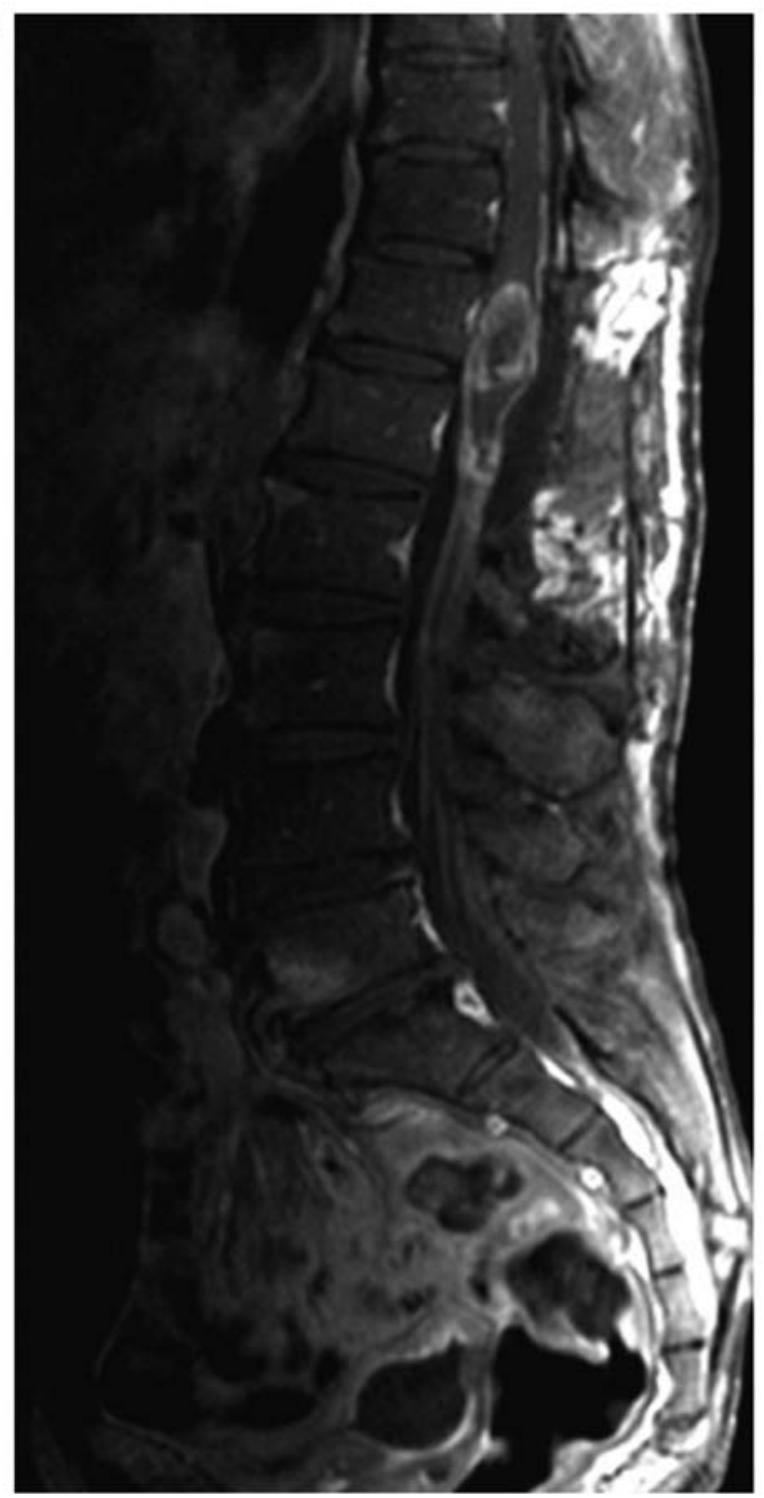

B

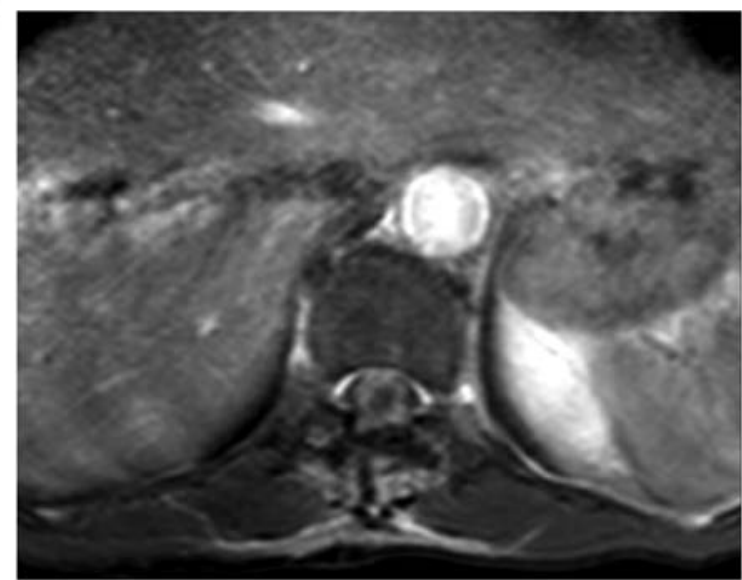

Figure 6. Postoperative enhancement. (A) Sagittal section and (B) cross-section. MRI did not detect the existence of a remaining tumour, soft tissue swelling was presented after surgery.

(2\%) (7). The femur is affected the most frequently, especially in the midshaft. However, ES is rarely observed in the spinal canal. Typically, the tumour consists of small circular cells with

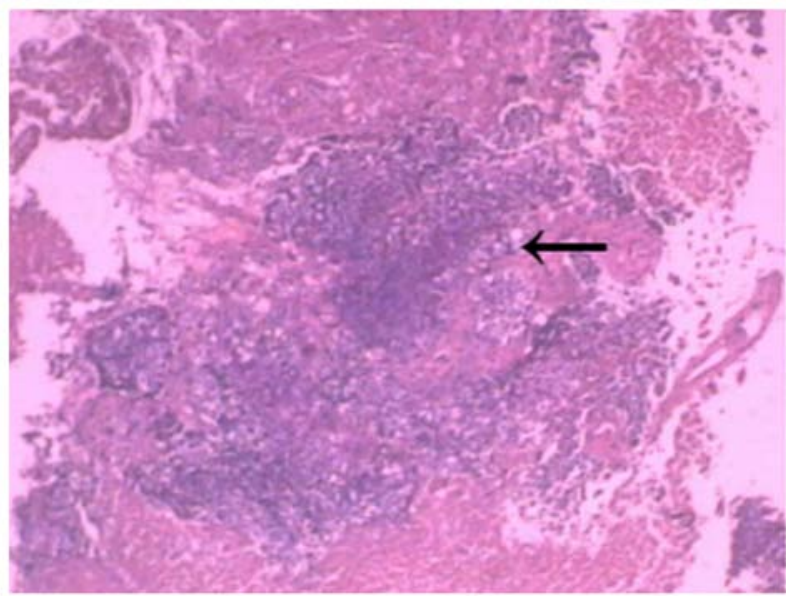

Figure 7. Haematoxylin and eosin staining. Hypercellular areas, monomorphic small circular blue cells lacking a cytoplasm with the focal clearing of the cytoplasm arranged in sheets and compact nest patterns were observed during histopathological analysis (magnification, x100). The arrow indicates that the positive product expression site is placed in the center of the field of view.

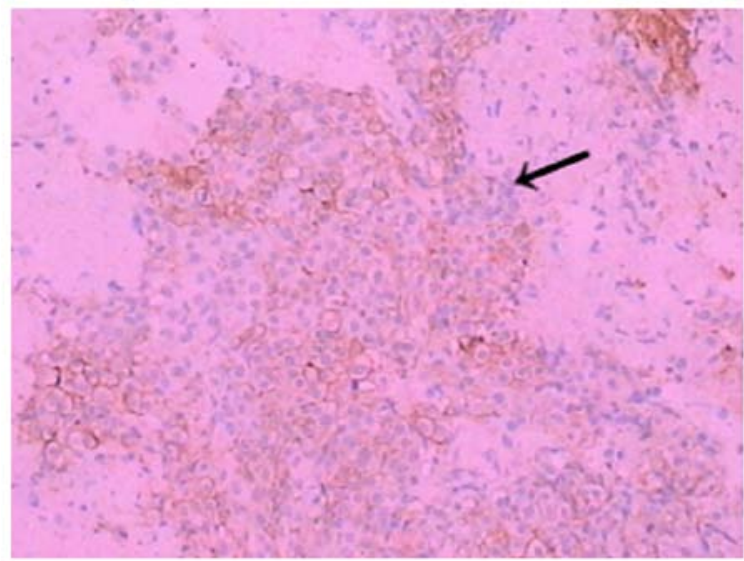

Figure 8. Immunohistochemical staining demonstrating that the tumour cells were positive for cluster of differentiation 99 (magnification, x400). The arrow indicates that the positive product expression site is placed in the center of the field of view.

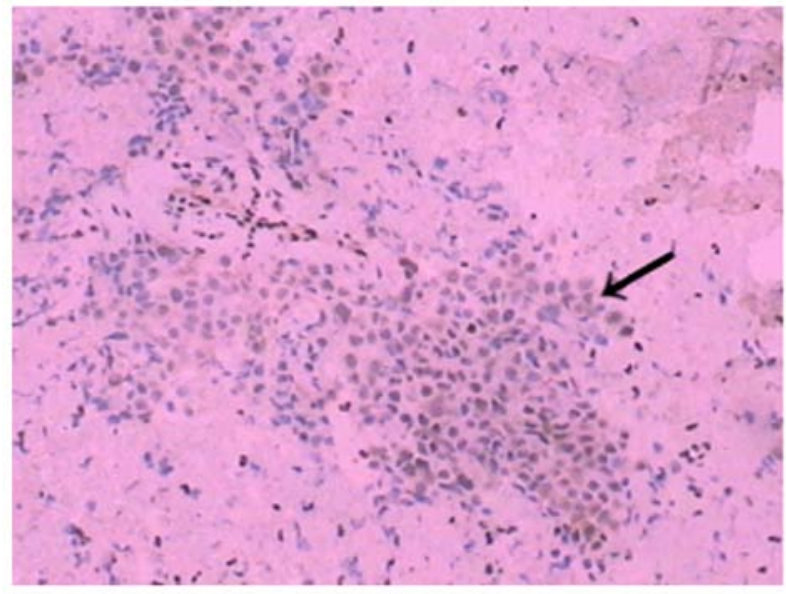

Figure 9. Immunohistochemical staining demonstrating that the tumour cells were positive for foetal-liver infusion 1 (magnification, $\mathrm{x} 400$ ). The arrow indicates that the positive product expression site is placed in the center of the field of view. 


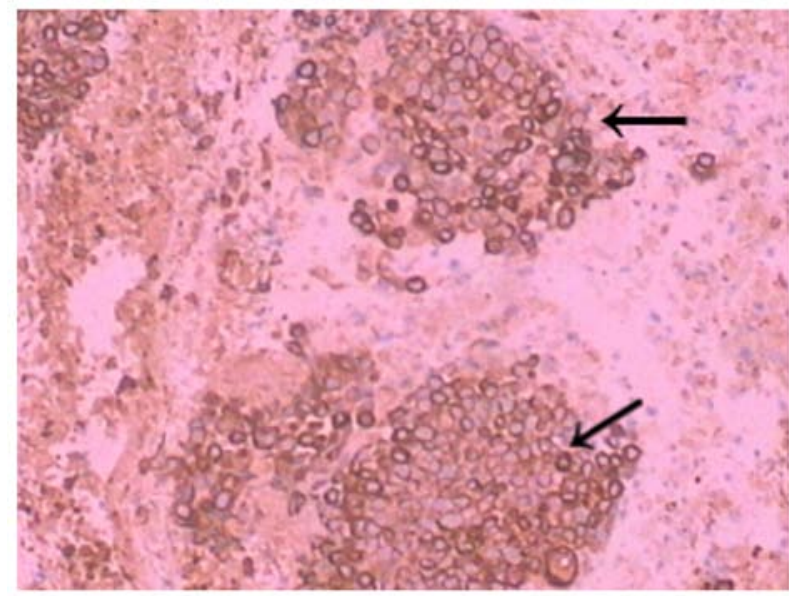

Figure 10. Immunohistochemical staining demonstrating that the tumour cells were positive for cytokeratin (magnification, $\mathrm{x} 400$ ).

regular circular nuclei containing finely dispersed chromatin and inconspicuous nucleoli, as well as a narrow rim of a clear or pale cytoplasm, which can be observed by light microscopy (2). Ultrastructural examination demonstrates that the tumour includes primitive cells with a smooth nuclear surface, scanty organelles and cytoplasmic glycogen in pools or aggregates (8). Tumours with similar histology also arise in soft tissues, such as peripheral primitive neuroectodermal tumours, neuroepitheliomas and Askin tumours (8). Pain is the most common symptom in patients with ES. Usually, the disease is concealed, and the pain may exist for a long time before the patient seeks medical treatment. Initial pain may be mild and intermittent and may respond to nonsurgical treatment (8). The average delay between symptom onset and diagnosis is 34 weeks. According to a previous report, the average time is 15 weeks between symptom onset and the first visit, and 19 weeks between the initial visit and a correct diagnosis (8). If the patient continues to experience symptoms, a radiographic examination is important as it can identify the primary lesion, when first diagnosed and during follow-up. In addition to pain, patients may experience fever, erythema and swelling (9). Laboratory tests may reveal an increase in the white blood cell count, the red blood cell rate (erythrocyte sedimentation rate) and C-reactive protein, which may be misdiagnosed as osteomyelitis (9). The patient described in the present case report was a 60 -year-old male patient who admitted to hospital following a surgical pathology diagnosis. Clinical literature attributes this type of pathology to rare diseases (10), and a limited number of reports of ES in the spine have been published $(10,11)$. ES is rare not only in middle-aged patients, but also in the spinal canal. In the present case, no tumours were identified in the spinal cord or vertebrae; they were distributed in the spinal canal, and the patient's EWSR1 FISH test was negative. No previous reports describing similar symptoms, diagnosis and complications were found in published literature. The diagnosis is further complicated by the similarity of the biopsy results of ES to those of pus (11), and the tissue may be sent to the microbiology department instead of the pathology department; the majority of biopsy specimens should be sent for bacterial culture and pathology. A number of common immunohistochemical markers expressed in ES, such as CD99, FLI-1 and CK, provide valuable support for ES
A

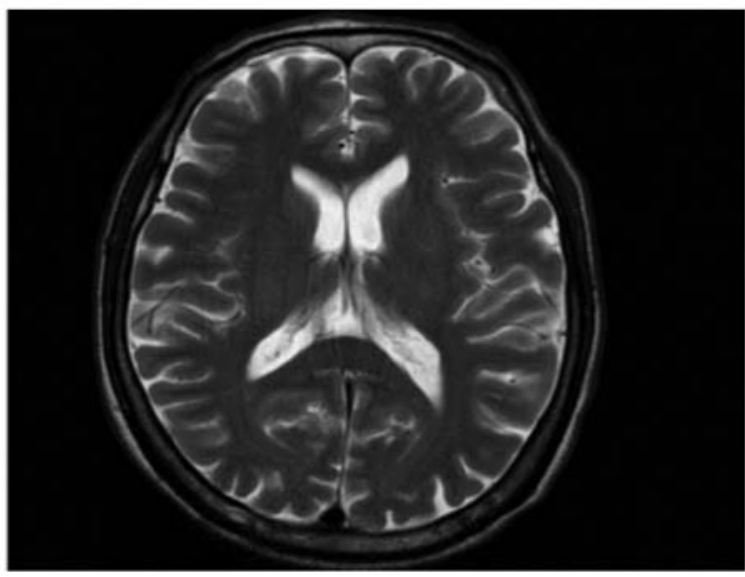

B

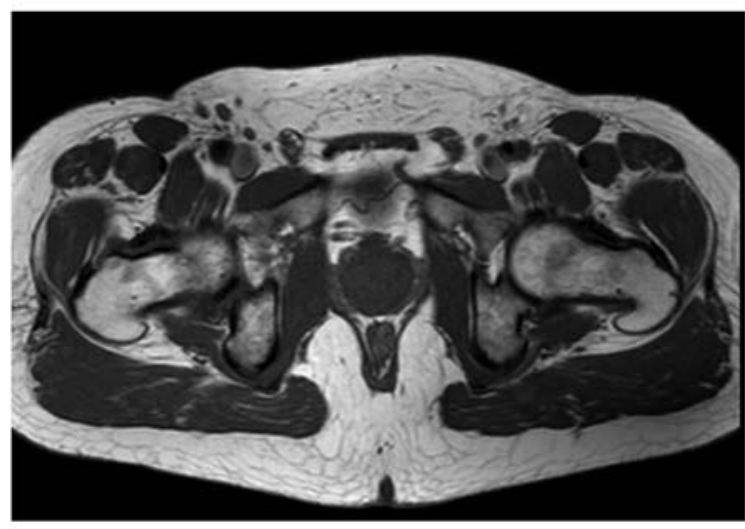

C

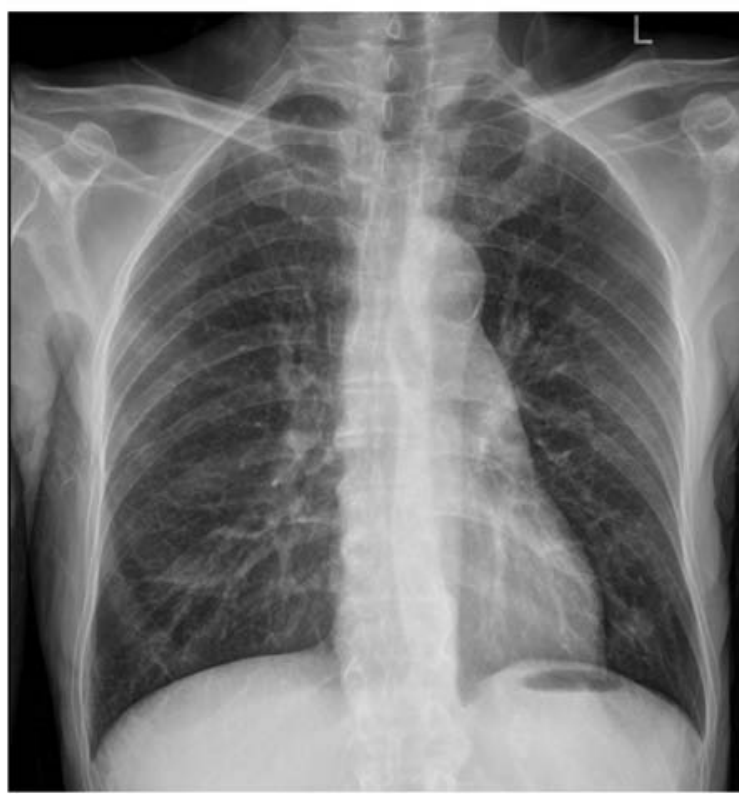

Figure 11. No suspicious lesions were detected in the patient's (A) brain, (B) prostate and (C) lungs.

diagnosis. CD99 is a $32 \mathrm{kDa}$ glycoprotein encoded by the MIC2 gene, and is used to diagnose ES with high sensitivity $(11,12)$; the sensitivity is $95 \%$, although the specificity is low (12-14). According to a previous report by Vural et al (14), FLI-1 expression was detected in 7/8 (87.5\%) ES cases, and CD99 expression was detected in 10/11 (90\%) ES cases. CD99 is the most sensitive immunohistochemical marker for ES. However, the expression of these markers has also been described in T-lymphoblastic 


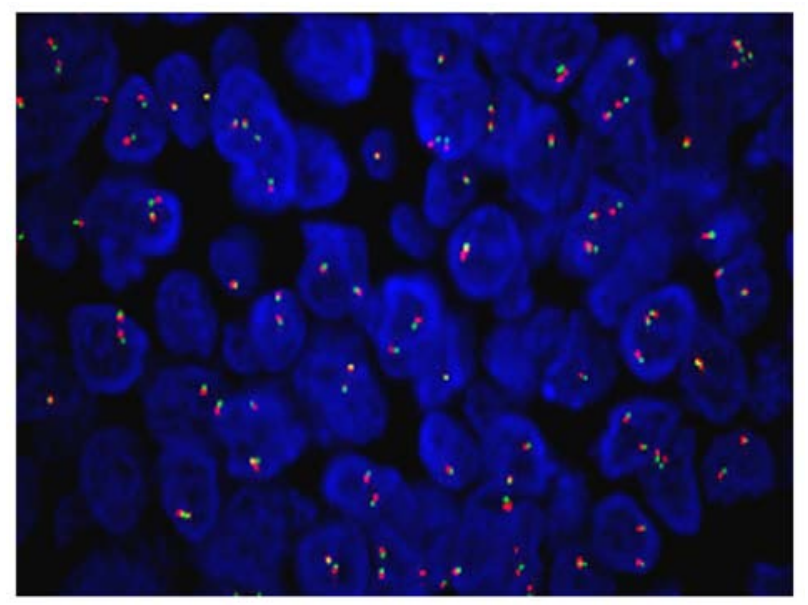

Figure 12. Genetic fluorescence in situ hybridization analysis. The Ewing's sarcoma breakpoint region 1gene exhibited no specific cytogenetic abnormalities after analysing 200 interphase cells.

lymphoma, rhabdomyosarcoma, synovial sarcoma and small cell anaplastic osteosarcoma (15-18). Therefore, ES may be misdiagnosed if based solely on the expression of CD99. FLI-1, as well as CK, is sensitive but less specific for ES compared with CD99 (11,13). Previous studies have demonstrated (19-25) that both markers are expressed in various other round cell tumours (19). The immunohistochemistry results of at least CD99 and FLI-1 are primarily markers of high specificity in the diagnosis of ES (20-22).

EWSR 1 is the most commonly translocated gene in sarcoma and is associated with a variety of mesenchymal lesions, such as ES, proliferative small circle cell tumour, clear cell sarcoma, vascular-like fibres, histiocytoma, extramedullary mucinous chondrosarcoma and mucinous liposarcoma (23-25). The detection of genetic FISH showed rupture abnormalities of the EWSR1 gene can assist in the differential diagnosis of ES and peripheral primitive neuroectodermal tumours; however, positivity does not necessarily indicate ES, and not all cases of ES are EWSR1-positive, which suggests that EWSR1 is not specific to ES $(26,27)$. EWSR1 rearrangement can be visualized by FISH; as soft tissue ES is diagnostically challenging, FISH analysis is a useful confirmatory diagnostic tool (28). However, as in the majority of instances in which a split-apart approach is used, the results of molecular genetics must be evaluated in the context of morphology. The patient described in the present report was subjected to FISH analysis to detect a fusion partner, but no rearrangements were identified. According to the presence of the EWSR1 gene rearrangement in only one of the three carcinomas (Ewing's sarcoma and hidradenoma of the skin and mucoepidermoid carcinoma of the salivary glands) studied by Möller et al (28), it may be concluded that the patient described in the present study was negative for EWSR1 gene rearrangement, suggesting that other mechanisms may be involved in the pathogenesis of this tumour type. This case was sent to Professor Anjia Han at the Department of Pathology of the First Affiliated Hospital of Sun Yat-sen University (Guangzhou, China) and other professors, who considered the lesion to be a malignant tumour, but did not exclude ES or metastatic cancer. Therefore, this case may be described as an EWSR1-negative ES in the primary spinal canal. Surgical treatment of tumours in the spine, pelvis or even the spinal canal is controversial (29). In addition to surgery and chemotherapy, several novel molecular targets for ES treatment have recently been identified and investigated in preclinical and clinical settings; treatments targeting the function of receptor tyrosine kinases, fusion protein EWSR1 and mTOR have demonstrated promising results (30). There has also been an increasing interest in the immune responses of patients with ES; immunotherapies using T cells, NK cells, cancer vaccines and monoclonal antibodies have been considered for ES, especially for recurrent cases (30). In the patient described in the present report, due to the sudden occurrence of spinal cone constriction and following multidisciplinary consultation, as well as consultation with the patient and his family, it was decided to immediately perform surgery to remove the tumour mass and relieve the spinal cord compression. The median survival time of patients with all stages of ES is 26.14 months, and the median survival of patients in the late metastatic stage is 5.6 months (31); although no randomized studies of ES occurrence in the spinal canal have been published, ifosfamide-based chemotherapy may have a positive impact on overall survival (32). A multidrug chemotherapy regimen for bone ES treatment can be administered; however, different responses to chemotherapy regimens have been observed. The most effective drugs include vincristine, actinomycin D, high-dose cyclophosphamide, doxorubicin, etoposide and ifosfamide (32). In the present case, radical surgery was performed, followed by the administration of combination chemotherapy, but the disease continued to progress. Thus, the best solution for each patient may be established following extensive discussions with the patient and his/her family.

In conclusion, the present report described for the first time a rare case of a Chinese patient with localized ES occurring in the spinal canal, as confirmed by genetic and ultrastructure analyses. This case and previous reports have revealed that surgery combined with chemotherapy and radiotherapy may contribute to significant improvements in survival; however, for each patient, the best treatment plan can be established through discussions with the patient and his/her family.

\section{Acknowledgements}

The authors would like to thank Professor Anjia Han from the Department of Pathology of the First Affiliated Hospital of Sun Yat-sen University (Guangzhou, China).

\section{Funding}

No funding was received.

\section{Availability of data and materials}

All data generated or analyzed during this study are included in this published article.

\section{Authors' contributions}

DZ conceived the study. DY acquired the data and performed the literature search. DY and JZ designed the study and analysed the data. DY and DZ prepared and edited the manuscript. All authors read and approved the final manuscript. 


\section{Ethics approval and consent to participate}

This study was approved by the Ethical Committee of the First Affiliated Hospital of Guangdong Pharmaceutics University (Guangzhou, China). The study conformed to the relevant regulatory standards.

\section{Patient consent for publication}

Written informed consent was obtained from the patient for this study. All patient identifiers have been removed.

\section{Competing interests}

The authors state that they have no competing interests.

\section{References}

1. Zucman J, Delattre O, Desmaze C, Plougastel B, Joubert I, Melot T, Peter M, De Jong P, Rouleau G, Aurias A, et al: Cloning and characterization of the Ewing's sarcoma and peripheral neuroepithelioma $t(11 ; 22)$ translocation breakpoints. Genes Chromosomes Cancer 5: 271-277, 1992.

2. Haresh KP,Chinikkatti SK, Prabhakar R, Rishi A, Rath GK, Sharma DN and Julka PK: A rare ease of intradural extramedullary Ewing's sarcoma with skip metastasis in the spine. Spinal Cord 46: 582-584, 2008.

3. Gardner LJ, Ayala AG, Monforte HL and Dunphy CH: Ewing sarcoma/peripheral primitive neuroectodermal tumor adult abdominal tumors with an Ewing sarcoma gene rearrangement demonstrated by fluorescence in situ hybridization in paraffin sections. Appl Immunohistochem Mol Morphol 12: 160-165, 2004

4. Ramos-Vara JA and Miller MA: When tissue antigens and antibodies get along: Revisiting the technical aspects of immunohistochemistry-the red, brown, and blue technique. Vet Pathol 51: 42-87, 2014.

5. Pernthaler A, Pernthaler J and Amann R: Fluorescence in situ hybridization and catalyzed reporter deposition for the identification of marine bacteria. Appl Environ Microbiol 68: 3094-3101, 2002.

6. Wagner M, Horn M and Daims H: Fluorescence in situ hybridisation for the identification and characterisation of prokaryotes. Curr Opin Microbiol 6: 302-309, 2003.

7. Grier HE: The Ewing family of tumors. Ewing's sarcoma and primitive neuroectodermal tumors. Pediatr Clin North Am 44: 991-1104, 1997.

8. Burchill SA: Ewing's sarcoma: Diagnostic, prognostic, and therapeutic implications of molecular abnormalities. J Clin Pathol 56: 96-102, 2003.

9. Iwamoto Y: Diagnosis and treatment of Ewing's sarcoma. Jpn J Clin Oncol 37: 79-89, 2007.

10. Ozturk E, Mutlu H, Sonmez G, Vardar Aker F, Cinar Basekim C and Kizilkaya E: Spinal epidural extraskeletal Ewing sarcoma. J Neuroradiol 34: 63-67, 2007.

11. Hung YP, Fletcher CD and Hornick JL: Evaluation of NKX2-2 expression in round cell sarcomas and other tumors with EWSR1 rearrangement: Imperfect specificity for Ewing sarcoma. Mod Pathol 29: 370-380, 2016

12. Ahmed AA, Nava VE, Pham T, Taubenberger JK, Lichy JH, Sorbara L, Raffeld M, Mackall CL and Tsokos M: Ewing sarcoma family of tumors in unusual sites: Confirmation by rt-PCR. Pediatr Dev Pathol 9: 488-495, 2006.

13. Llombart-Bosch A, Machado I, Navarro S, Bertoni F, Bacchini P, Alberghini M, Karzeladze A, Savelov N, Petrov S, Alvarado-Cabrero I, et al: Histological heterogeneity of Ewing's sarcoma/PNET: An immunohistochemical analysis of 415 genetically confirmed cases with clinical support. Virchows Arch 455: 397-411, 2009.

14. Vural C, Uluoğlu O, Akyürek N, Oğuz A and Karadeniz C: The evaluation of CD99 immunoreactivity and EWS/FLI1 translocation by fluorescence in situ hybridization in central PNETs and Ewing's sarcoma family of tumors. Pathol Oncol Res 17: 619-625, 2011.

15. Ambros IM, Ambros PF, Strehl S, Kovar H, Gadner H and Salzer-kuntschik M: MIC2 is a specific marker for Ewing's-sarcoma and peripheral primitive neuroectodermal tumors. Evidence for a common histogenesis of Ewing's sarcoma and peripheral primitive neuroectodermal tumors from MIC2 expression and specific chromosome aberration. Cancer 67: 1886-1893, 1991.
16. Gerald WL, Ladanyi M, de Alava E, Cuatrecasas M, Kushner BH, LaQuaglia MP and Rosai J: Clinical, pathologic, and molecular spectrum of tumors associated with $\mathrm{t}(11 ; 22)$ (p13;q12): Desmoplastic small round-cell tumor and its variants. J Clin Oncol 16: 3028-3036, 1998.

17. Devaney K, Vinh TN and Sweet DE: Small-cell osteosarcoma of bone: An immunohistochemical study with differential diagnostic considerations. Hum Pathol 24: 1211-1225, 1993.

18. Riopel M, Dickman PS, Link MP and Perlman EJ: MIC2 analysis in pediatric lymphomas and leukemias. Hum Pathol 25: 396-399, 1994.

19. Mhawech-Fauceglia P, Herrmann FR, Bshara W, Odunsi K, Terracciano L, Sauter G, Cheney RT, Groth J, Penetrante R and Mhawech-Fauceglia P: Friend leukaemia integration-1 expression in malignant and benign tumours: A multiple tumour tissue microarray analysis using polyclonal antibody. J Clin Pathol 60: 694-700, 2007.

20. Saxena R, Sait S and Mhawech-Fauceglia P: Ewing sarcoma/primitive neuroectodermal tumor of the kidney: A case report. Diagnosed by immunohistochemistry and molecular analysis. Ann Diagn Pathol 10: 363-366, 2006.

21. Zhong J, Chen N, Chen X, Gong J, Nie L, Xu M and Zhou Q: Peripheral primitive neuroectodermal tumor of the kidney in a 51-year-old female following breast cancer: A case report and review of the literature. Oncol Lett 9: 108-112, 2015.

22. Pinto A, Dickman P and Parham D: Pathobiologic markers of the Ewing sarcoma family of tumors: State of the art and prediction of behaviour. Sarcoma 2011: 856190, 2011.

23. Mazur MA, Gururangan S, Bridge JA, Cummings TJ, Mukundan S, Fuchs H, Larrier N and Halperin EC: Intracranial Ewing sarcoma. Pediatr Blood Cancer 45: 850-856, 1999.

24. Llombart-Bosch A, Machado I, Navarro S, Bertoni F, Bacchini P, Alberghini M, Karzeladze A, Savelov N, Petrov S, Alvarado-Cabrero I, et al: Histological heterogeneity of Ewing's sarcoma/PNET: An immunohistochemical analysis of 415 genetically confirmed cases with clinical support. Virchows Arch 455: 397-411, 2009.

25. Weidner $\mathrm{N}$ and Tjoe J: Immunohistochemical profile of monoclonal antibody O13: Antibody that recognizes glycoprotein p30/32MIC2 and is useful in diagnosing Ewing's sarcoma and peripheral neuroepithelioma. Am J Surg Pathol 18: 486-494, 1994.

26. Lessnick SL and Ladanyi M: Molecular pathogenesis of Ewing sarcoma: New therapeutic and transcriptional targets. Annu Rev Pathol 7: 145-159, 2012.

27. Romeo S and Dei Tos AP: Soft tissue tumors associated with EWSR1 translocation. Virchows Arch 456: 219-234, 2010.

28. Möller E, Stenman G, Mandahl N, Hamberg H, Mölne L, van den Oord JJ, Brosjö O, Mertens F and Panagopoulos I: POU5F1, encoding a key regulator of stem cell pluripotency, is fused to EWSR1 in hidradenoma of the skin and mucoepidermoid carcinoma of the salivary glands. J Pathol 215: 78-86, 2008.

29. Sankar S, Bell R, Stephens B, Zhuo R, Sharma S, Bearss DJ and Lessnick SL: Mechanism and relevance of EWS/FLI-mediated transcriptional repression in Ewing sarcoma. Oncogene 32: 5089-5100, 2013.

30. Naing A, LoRusso P, Fu S, Hong DS, Anderson P, Benjamin RS, Ludwig J, Chen HX, Doyle LA and Kurzrock R: Insulin growth factor-receptor (IGF-1R) antibody cixutumumab combined with the mTOR inhibitor temsirolimus in patients with refractory Ewing's sarcoma family tumors. Clin Cancer Res 18: 2625-2631, 2012.

31. Ladenstein R, Pötschger U, Le Deley MC, Whelan J,Paulussen M, Oberlin O, van den Berg H, Dirksen U, Hjorth L, Michon J, et al: Primary disseminated multifocal Ewing sarcoma: Results of the Euro-EWING 99 trial. J Clin Oncol 28: 3284-3291, 2010.

32. Grier HE, Krailo MD, Tarbell NJ,Link MP, Fryer CJ,Pritchard DJ, Gebhardt MC, Dickman PS, Perlman EJ, Meyers PA, et al: Addition of ifosfamide and etoposide to standard chemotherapy for Ewing's sarcoma and primitive neuroectodermal tumor of bone. N Engl J Med 348: 694-701, 2003.

(i) $\ominus$ This work is licensed under a Creative Commons Attribution-NonCommercial-NoDerivatives 4.0 International (CC BY-NC-ND 4.0) License. 